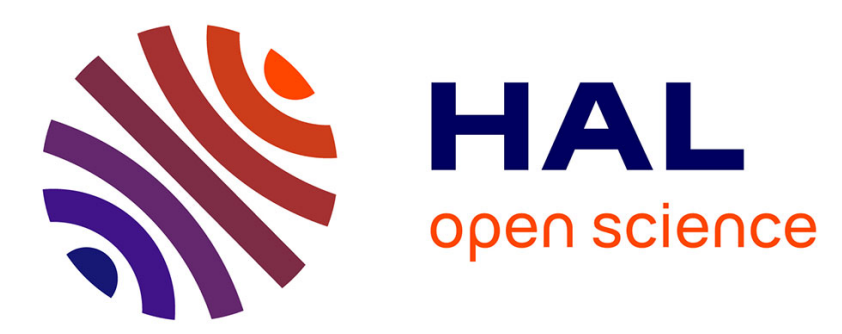

\title{
Dynamics of ethane and propane in zeolite ZSM-5 studied by quasi-elastic neutron scattering
}

Hervé Jobic, Marc Bee, Gordon Kearley

\section{To cite this version:}

Hervé Jobic, Marc Bee, Gordon Kearley. Dynamics of ethane and propane in zeolite ZSM-5 studied by quasi-elastic neutron scattering. Zeolites, 1992, 12, pp.146-151. 10.1016/0144-2449(92)90075-Z . hal-00006264

\section{HAL Id: hal-00006264 \\ https://hal.science/hal-00006264}

Submitted on 10 Jan 2022

HAL is a multi-disciplinary open access archive for the deposit and dissemination of scientific research documents, whether they are published or not. The documents may come from teaching and research institutions in France or abroad, or from public or private research centers.
L'archive ouverte pluridisciplinaire HAL, est destinée au dépôt et à la diffusion de documents scientifiques de niveau recherche, publiés ou non, émanant des établissements d'enseignement et de recherche français ou étrangers, des laboratoires publics ou privés.

\section{(ㄷ)(1) $\$$}

Distributed under a Creative Commons Attribution - NonCommercial| 4.0 International 


\title{
Dynamics of ethane and propane in zeolite ZSM-5 studied by quasi-elastic neutron scattering
}

\author{
Hervé Jobic \\ Institut de Recherches sur la Catalyse, Villeurbanne Cedex, France \\ Marc Bée \\ Laboratoire de Spectrométrie Physique, Université Joseph Fourier, St Martin d'Hères, France \\ Gordon J. Kearley \\ Institut Laue-Langevin, Grenoble, France
}

\begin{abstract}
Quasi-elastic neutron scattering has been used to study the dynamics of ethane and propane in zeolite ZSM-5. The experiments were performed at different loadings for the two alkanes and at different temperatures for propane. The long-range translational motion of the molecules has been observed and has been interpreted with a jump diffusion model, the mean jump lengths being of the order of 10 $\AA$, slightly decreasing with increasing loading. The self-diffusion coefficients of ethane are about $2 \times$ $10^{-9} \mathrm{~m}^{2} \mathrm{~s}^{-1}$ at $300 \mathrm{~K}$, those of propane at the same temperature being smaller by a factor of two. An activation energy of $5 \mathrm{~kJ} \mathrm{~mol}^{-1}$ is obtained for the self-diffusion of propane. These results are in good agreement with the pulsed-field gradient n.m.r. measurements. In addition to the translational motion, a rotational motion of the two molecules is also observed; it is described by an uniaxial rotational diffusion model and rotational diffusion constants are derived.
\end{abstract}

Konwords: Diffusion; ethane; propane; ZSM-5; quasi-elastic neutron scattering

\section{INTRODUCTION}

The main applications of ZSM-5-class zeolites are found in the field of chemical and fuel processing and are based on the shape-selective properties of its structure. The framework of ZSM-5 consists of straight channels interconnected by sinusoidal channels, each made of 10 -membered oxygen rings. ${ }^{1}$ These channels exhibit sizes (free diameter ca. $5.5 \AA$ ) similar to simple aromatics and isoparaffins.

Diffusion coefficients of molecules in ZSM-5-type zeolites (including silicalite, the purely siliceous polymorph of ZSM-5) are currently being measured by a large number of techniques. However, the reported diffusion coefficients for a given molecule may differ by six orders of magnitude. ${ }^{2}$ The greatest differences are usually observed between the microscopic methods: pulsed-field gradient n.m.r. and quasielastic neutron scattering (QENS), and the macroscopic methods: gravimetry, chromatography, etc. New experimental methods have been recently developed; for example, the frequency-response, either in its single-step version or not $_{2}{ }^{3,4}$ gives results close to those of the microscopic techniques.
In the last few years, molecular dynamics (MD) simulations have also been used to investigate the diffusion of noble gases or small hydrocarbons in silicalite. $^{5-9}$ The self-diffusion coefficients of methane computed from the trajectories created by the MD simulations were found to be in good agreement with the n.m.r. and QENS results., ${ }^{5,10}$

Because of experimental limitations, the range of diffusion coefficients that can be measured by n.m.r. and QENS is restricted. For example, the mobility of benzene in ZSM-5 has recently been found to be below the limit of measurability of the two techniques: because of the short transverse relaxation time $T_{2}$ of the adsorbed molecule in n.m.r. ${ }^{11}$ and because of the instrumental resolution limit in QENS. ${ }^{12}$ For the same reason, only the diffusion of short-chainlength paraffins (up to propane) has been measured by n.m.r. ${ }^{13}$ In the case of methane adsorbed at different loadings and temperatures in ZSM-5, an excellent agreement has been observed for the selfdiffusion coefficients, for the mean jump lengths, and for the activation energy determined by n.m.r. and QENS. ${ }^{2}$

We report in this paper QENS results on the translational and rotational dynamics of ethane and 
propane adsorbed at different loadings in NaZSM-5. Self-diffusion coefficients and mean jump lengths are derived and compared with those obtained by n.m.r. ${ }^{13}$ and also by frequency response, ${ }^{3,4}$ membrane, ${ }^{14}$ and zero-length column ${ }^{15}$ techniques. For propane, QENS experiments have been performed at different temperatures so that an activation energy for the self-diffusion is obtained.

\section{EXPERIMENTAL}

The experimental results were obtained at the Institut Laue-Langevin, Grenoble, using the time-of-flight (TOF) spectrometer IN5. The incident wavelength was taken as $9 \AA$ ( $1 \mathrm{meV})$, giving an elastic resolution of $\approx 18 \mu \mathrm{eV}$ (full-width at half-maximum). The Bragg peaks of the zeolite were determined on the spectrometer by comparing the intensities of the elastic peaks at each angular position with those obtained from a standard vanadium plate (vanadium is an almost totally incoherent scatterer and is used to calibrate the detector's efficiencies and to measure the elastic resolution). The 110 TOF spectra were grouped by the instrument's computer into several $Q$-space regions, avoiding the Bragg peaks of the zeolite (the neutron momentum transfer is $\hbar Q$ ). The spectra were recorded in transmission geometry by orienting the cell at $130^{\circ}$ to the incident beam.

The NaZSM-5 sample was the same as in our previous study on methane; ${ }^{16}$ several methods have been used for its characterization. ${ }^{2}$ Adsorption isotherms were measured with an electromagnetic balance (Sartorius), the pressure being monitored by a capacitance manometer (Leybold Inficon). The zeolite was treated in a glass reaction vessel at $700 \mathrm{~K}$ under flowing oxygen and outgassed to a final pressure of $10^{-3} \mathrm{~Pa}$ at the same temperature. The zeolite was then transferred, inside a glovebox, into two slab-shaped aluminum containers of circular geometry (diameter $50 \mathrm{~mm}$ ). The mass of the activated zeolite was $\approx 5 \mathrm{~g}$, giving a neutron transmission of $97 \%$ (for $\lambda=9 \AA$ ). The cells were connected to a gas inlet system allowing loading of the samples in situ with the hydrocarbons at different pressures and temperatures.

The TOF spectra were corrected for incident neutron flux, detector efficiency, absorption, and selfshielding. The scattering of the bare zeolites was subtracted from the spectra, and the TOF spectra were then transformed to an energy scale $(\hbar \omega)$.

\section{THEORY}

For hydrocarbons in zeolites, only incoherent scattering has to be considered because of the large incoherent cross section of hydrogen. ${ }^{17}$ The intensity scattered by the sample is proportional to the incoherent scattering law, $S_{\text {inc }}(Q, \omega)$, which is related to self-motions of the hydrogen atoms under the effect of the different molecular motions. The total scattering law is a convolution product of the individual scattering laws corresponding to the different motions of translation, rotation, and vibration.
Since no internal modes of the molecules are excited in the energy range studied (up to $1 \mathrm{meV}$ ), the contribution from the vibrational motions appears under the form of a flat background and of a Debye-Waller factor, $\exp \left(-Q^{2}<u^{2}>\right)$, where $<u^{2}>$ is the mean-square hydrogen amplitude due to the internal motions.

\section{Rotational scattering law}

For methane adsorbed in ZSM-5 (Ref. 16) and for ethane in $\mathrm{NaX}$ (Ref. 18), it was found that the QENS spectra were well described by an isotropic rotational diffusion model. ${ }^{19}$ For ethane in ZSM-5, this model was not able to reproduce satisfactorily the quasielastic profiles when fits were made on the whole spectra. We have therefore considered another model, less isotropic, in which the molecule performs continuous rotational diffusion àbout its main molecular axis; this model will also be used for longer alkanes (up to hexane). No analytical expression can be calculated for a powder sample, ${ }^{20}$ but very close approximations to structure factors can be derived by considering a jump model over $N$ equally spaced sites, with a sufficiently large number of sites. A number of jumps $\geqslant 6$ is found to be sufficient at low $Q$ values; this corresponds to the following scattering law:

$$
S_{\text {inc }}^{\text {rot }}(Q, \omega)=A_{0}(Q) \delta(\omega)+\frac{1}{\Pi} \sum_{m=1}^{5} A_{m}(Q) \frac{\tau_{m}}{1+\omega^{2} \tau_{m}^{2}}(1)
$$

The elastic $(m=0)$ and quasi-elastic structure factors are defined by:

$$
A_{m}(Q)=\frac{1}{6} \sum_{p=1}^{6} j_{0}\left(2 Q r \sin \left(\frac{\Pi_{p}}{6}\right)\right) \cos \left(\frac{2 \Pi p m}{6}\right),
$$

where $j_{0}$ is the spherical Bessel function of order zero, and $r$, the radius of gyration.

The half-widths at half-maximum (hwhm), $\tau_{m}^{-1}$, of the Lorentzian functions are related to the mean time $\tau$ between successive rotational jumps:

$$
\tau_{m}^{-1}=\frac{2}{\tau} \sin ^{2}\left(\frac{\Pi m}{6}\right) .
$$

With this model $(N=6)$, there are only three different Lorentzian widths and it is then possible to add together the coefficients $A_{m}(Q)$ related to the same value of $\tau_{m}^{-1}$. The variation with $Q$ of the elastic and quasi-elastic structure factors is shown in Figure 1 for a radius of gyration of $2 \AA$ (the reason to use this value will be given in the Discussion). It can be seen that at low $Q$ values, the main contribution to the quasi-elastic scattering comes from the $m=1$ term. A rotational-diffusion constant, $D_{r}$, can then be identified with the jump rate probability $1 / \tau_{1}$ given by Equation (3):

$$
D_{r}=\frac{1}{\tau_{1}}=\frac{1}{2 \tau} \text {. }
$$

\section{Translational scattering law}

Precise QENS measurements recently performed on the translational mobility of molecules adsorbed in 


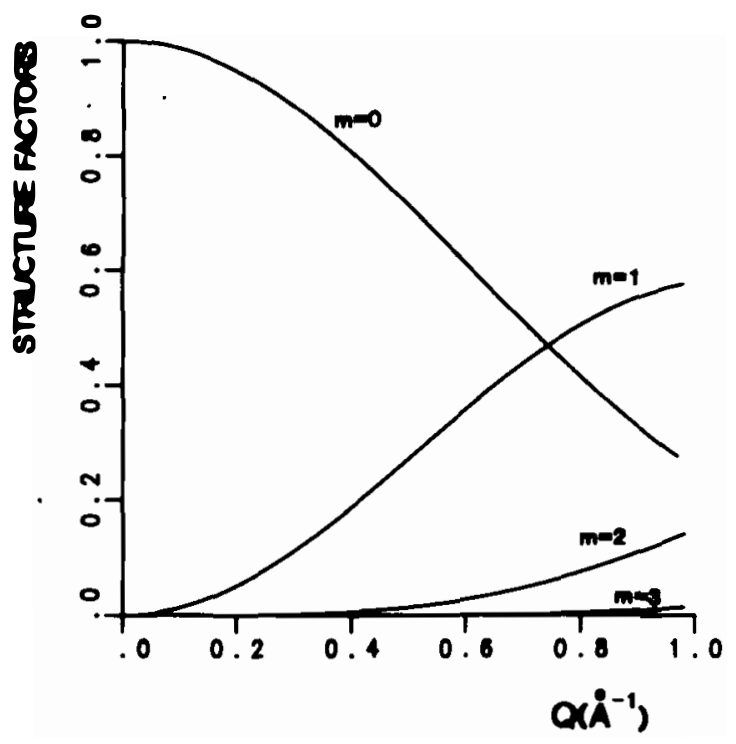

Figure 1 Elastic $(m=0)$ and quasi-elastic structure factors for an uniaxial rotational diffusion (radius of gyration: $2 \AA$ )

zeolites have been fitted by different jump diffusion processes. ${ }^{16,18,21,22}$ For ethane and propane adsorbed in ZSM-5, we find that the translational motion can be described by the jump diffusion model of Singwi and Sjölander (hereafter cited as S \& S). ${ }^{23}$ This model was originally written to characterize the diffusive motions in liquid water where the molecules are trapped by hydrogen bonds in pseudo-equilibrium positions, within a certain time interval, $\tau_{0}$, during which they perform a large number of oscillations; then they jump or diffuse during a mean time $\tau_{1}$ until they are trapped again in a new equilibrium position. This model can be applied to the diffusion of ethane and propane in ZSM-5 because the molecules perform low-frequency vibrations in given regions of space before they diffuse to another region in the channel network. Such limited spatial distributions have been calculated for different alkanes in silicalite via Monte Carlo techniques. ${ }^{24}$ An important assumption in the S \& $\mathrm{S}$ model is that no correlation exists between the motions in consecutive steps. This is justified if the molecule performs a large number of oscillations until it diffuses to another position. This is indeed the case for alkanes in silicalite where $\tau_{0}$ is of the order of $10^{-10} \mathrm{~s}$ and the period of the external vibrations is in the range $10^{-13}-10^{-12} \mathrm{s.}^{7,25}$ Moreover, when the molecule oscillates for a much longer time than it diffuses, $\tau_{0}>\tau_{1}$, the scattering law reduces to:

$$
S_{\text {inc }}^{\text {trans }}(Q, \omega)=\exp \left(-Q^{2}<\mathrm{d}^{2}>\right) \frac{1}{\Pi \omega^{2}+(\Delta \omega)^{2}} .
$$

The shape of the quasi-elastic peak is a Lorentzian function whose hwhm is given by:

$$
\Delta \omega=\frac{1}{\tau_{0}}\left[1-\frac{\exp \left(-Q^{2}<d^{2}>\right)}{1+D Q^{2} \tau_{0}}\right] .
$$

The actual self-diffusion coefficient, $D$, corresponds to:

$$
D=\frac{<1^{2}>+<d^{2}>}{6\left(\tau_{0}+\tau_{1}\right)}
$$

where $\left\langle 1^{2}\right\rangle$ is the mean-square jump distance, and $\left\langle d^{2}\right\rangle$, the mean-square radius of the fully developed thermal cloud in the oscillatory motion. Since $\tau_{1}<<$ $\tau_{0}$ and since the radius of the thermal cloud is found to be much smaller than the jump distance (v.i.), one gets the usual expression for the self-diffusion coefficient:

$$
D=\frac{<1^{2}>}{6 \tau_{0}} .
$$

At small $Q$ values, Equation (6) reduces to $\Delta \omega=D Q^{2}$. At high $Q$ values, the broadening approaches the asymptotic value: $1 / \tau_{0}$.

\section{RESULTS AND DISCUSSION}

\section{Ethane}

The dynamics of ethane in NaZSM- 5 were investigated at $300 \mathrm{~K}$ and at three different loadings: 1, 4, and 8 molecules/unit cell, on average. Greater loadings were not studied since these would contain multiple scattering effects. ${ }^{26}$

The spectra obtained at the different $Q$ values have been fitted simultaneously with the scattering law for uniaxial rotation convoluted with the $S$ \& $S$ model and with the instrumental resolution. Seven parameters are refined simultaneously: the self-diffusion coefficient, $D$; the mean residence time, $\tau_{0}$; the Debye-Waller factor; the radius of gyration, $r$; the rotational-diffusion constant, $D_{r}$; a flat background; and a scaling factor. Figure 2 shows a comparison between experimental and calculated energy spectra for a loading of 4 molecules/u.c., at different $Q$ values. The refined parameters are shown in Table 1 , the values of the mean jump lengths, $\left\langle 1^{2}\right\rangle^{1 / 2}$, being derived from Equation (8).

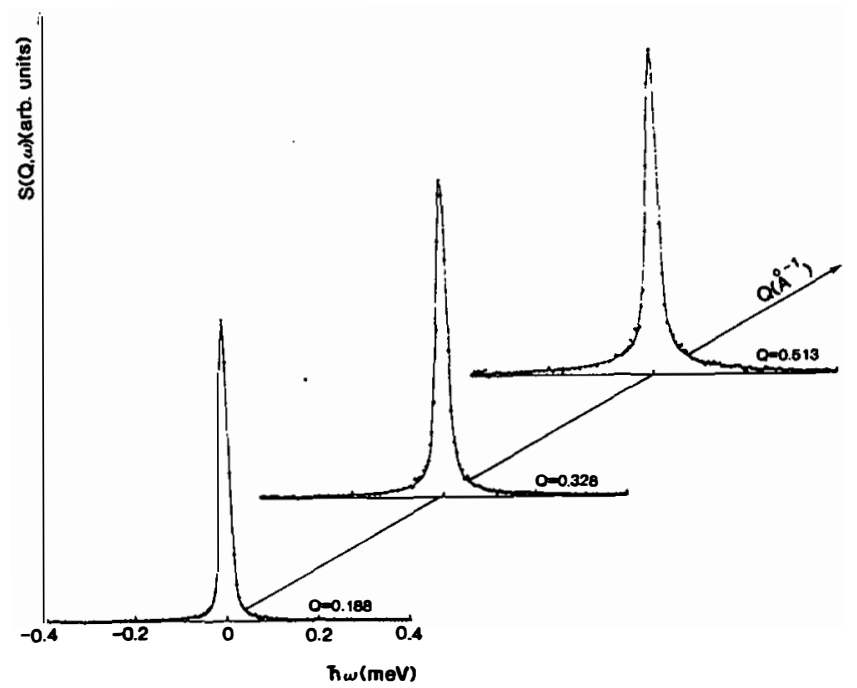

Figure 2 Quasi-elastic neutron spectra of ethane in ZSM-5 at different $Q$ values ( $T=300 \mathrm{~K}, 4$ molecules per u.c.) 


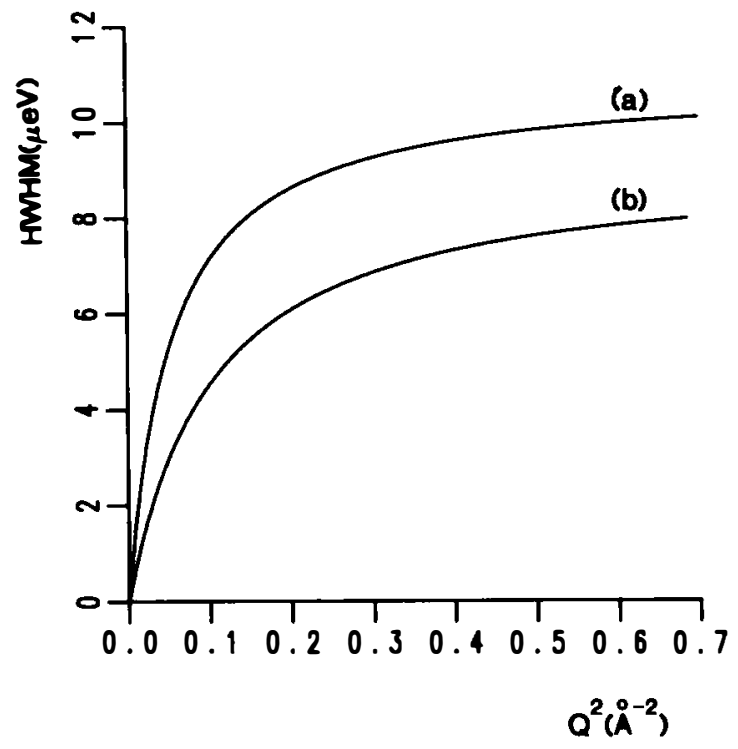

Figure 3 Broadening of the elastic peak v. $Q^{2}$ for ethane in ZSM-5 at $300 \mathrm{~K}$ : (a) 4 molecules per u.c.; (b) 8 molecules per u.c.

The broadenings of the elastic peak, Equation (6), are shown in Figure 3, versus $Q^{2}$, for two loadings. The values of the self-diffusion coefficients that are given in Table 1 are in good agreement with those obtained by pulsed-field gradient n.m.r., ${ }^{13}$ if one takes into account the experimental errors that are about $50 \%$ in each technique. Our results are also in agreement with the diffusion coefficients determined by the frequency-response method, ${ }^{3,4}$ but they are two orders of magnitude greater than those obtained with a molecular sieve membrane. ${ }^{14}$ There is also agreement on the mean jump lengths derived from the two microscopic methods: It is of the order of 10 $\AA$ at low loading, slightly decreasing with increasing loading. ${ }^{13}$ It is clear that the mean jump lengths for ethane in ZSM-5 are larger than those obtained for the same molecule in NaX zeolite. ${ }^{18}$

The mean residence time, $\tau_{0}$, is of the order of $10^{-10} \mathrm{~s}$ (Table 1 ), which means that the $\mathrm{S} \& \mathrm{~S}$ model can be applied, since this is much larger than the period of the external vibrations that is found to be in the range $10^{-13}-10^{12} \mathrm{~s}$.

Only one Debye-Waller factor was fitted to the data; this is justified by the large values of the

Table 1 Values of the parameters for the translational and rotational motions of ethane adsorbed at different loadings in NaZSM-5, at $300 \mathrm{~K}$ (values in parentheses correspond to n.m.r. results ${ }^{13}$ )

\begin{tabular}{lccl}
\hline & 1 molecule/u.c. & 4 molecules/u.c. & 8 molecules/u.c. \\
\hline$D\left(\mathrm{~m}^{2} \mathrm{~s}^{-1}\right)$ & $2 \times 10^{-9}$ & $3 \times 10^{-9}$ & $1.25 \times 10^{-9}$ \\
& & $\left(3.8 \times 10^{-9}\right)$ & $\left(2.3 \times 10^{-9}\right)$ \\
$\tau_{0}(\mathrm{~s})$ & $10^{-10}$ & $0.62 \times 10^{-10}$ & $0.75 \times 10^{-10}$ \\
$\left\langle\left.\right|^{2}\right\rangle^{1 / 2}(\AA)$ & 11 & 10.5 & 7.5 \\
$\left\langle d^{2}\right\rangle\left(\AA^{2}\right)$ & 1.25 & 0.66 & 0.63 \\
$r(\AA)$ & 3 & 2.4 & 2 \\
$D_{r}\left(\mathrm{~s}^{-1}\right)$ & $0.9 \times 10^{11}$ & $1.85 \times 10^{11}$ & $1.8 \times 10^{11}$ \\
\hline
\end{tabular}

mean-square radii of the thermal clouds, $\left\langle d^{2}\right\rangle$, relative to the mean-square amplitude due to the internal motions, $\left.<u^{2}\right\rangle$ (a value of $0.012 \AA^{2}$ was derived for $\left\langle u^{2}\right\rangle$ using a standard force field for hydrocarbons ${ }^{27}$ ). It can also be seen in Table 1 that the criterium of the $\mathrm{S} \& \mathrm{~S}$ model; $\left.\left.<d^{2}\right\rangle<<<1^{2}\right\rangle$, is fulfilled in our case.

The radii of gyration, $r$, obtained for the uniaxial rotation of ethane vary between 2 and $3 \AA$, which indicates that the position of the $\mathrm{C}-\mathrm{C}$ axis changes during the oscillatory state; the rotational-diffusion constants, $D_{r}$, are of the order of $10^{11} \mathrm{~s}^{-1}$. However, these should only be regarded as "effective" rotational-diffusion constants since (i) the radii of gyration are different from the one about a fixed axis and (ii) the rotational model that we use might be too simple to describe the details of the motion over small distances.

\section{Propane}

Similar results have been obtained for propane adsorbed at $300 \mathrm{~K}$ in NaZSM-5 for different loadings: $1.5,4$, and 8 molecules/u.c. Further experiments were performed at different temperatures for a fixed loading of 3 molecules/u.c. (by varying the equilibrium pressure), in order to get the activation energy for the diffusion of propane.

The models used for the rotational and translational motions of ethane were also used for propane. Examples of the fits between experimental and calculated spectra are shown in Figure 4 for a loading of 8 molecules/u.c., at $300 \mathrm{~K}$. The values of the refined parameters are given in Table 2 for the three propane loadings at $300 \mathrm{~K}$.

The broadening of the elastic peak versus $Q^{2}$ is shown in Figure 5 for a loading of 8 molecules/u.c. at $300 \mathrm{~K}$; the points correspond to individual fits of the spectra. When the fits are made simultaneously with all spectra, the parameters given in Table 2 are obtained.

Since we are interested mainly in the determination

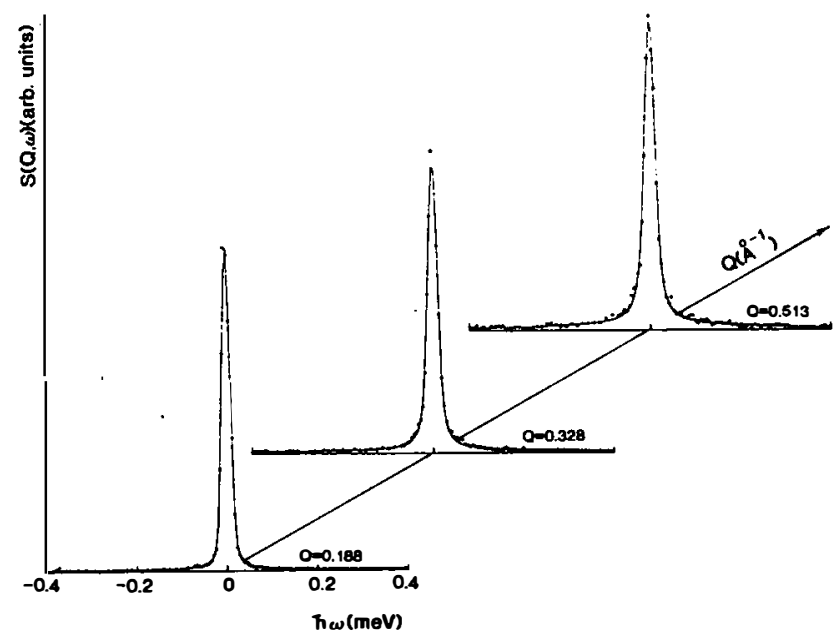

Figure 4 Energy spectra for propane in ZSM-5, at different $Q$ values ( $T=300 \mathrm{~K}, 8$ molecules per u.c.) 


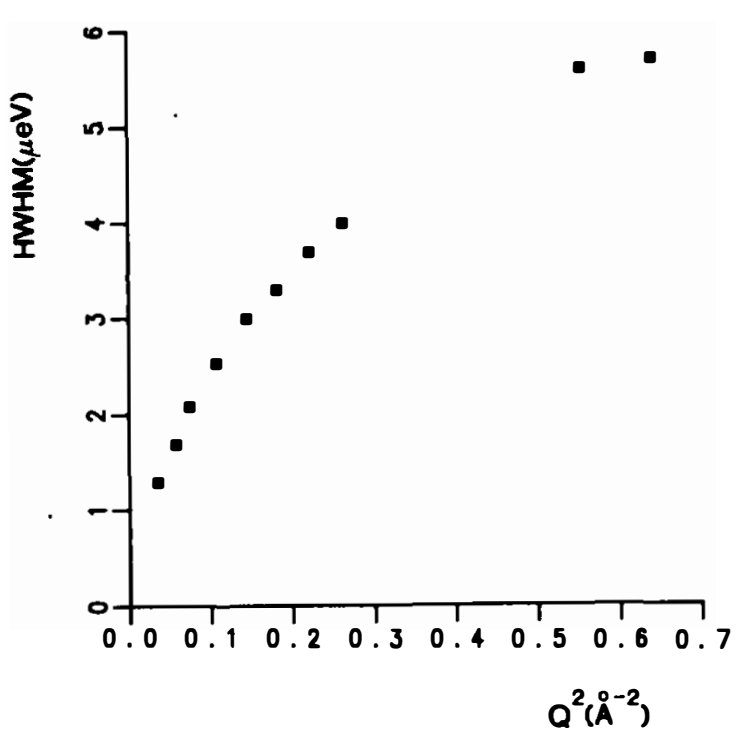

Figure 5 Broadening of the elastic peak v. $Q^{2}$ for propane in ZSM-5 at $300 \mathrm{~K}$ ( 8 molecules per u.c.); the points correspond to individual fits of the spectra

of diffusion coefficients, the small $Q$ range, corresponding to diffusion over a few unit cells, is the most important region. It is worth noting that in this $Q$ range the shape of the quasi-elastic peak is simply a Lorentzian function whose width is independent of the model, $\Delta \omega=D Q^{2}$. It can be seen in Table 2 (and also in Table 1) that the self-diffusion coefficient is smaller at the lowest loading. This could be due to a larger experimental error, since the increase of signal due to the hydrocarbon is only $\approx 20 \%$ for ethane and $\approx 40 \%$ for propane, at this loading. This could also be due to the trapping of a significant proportion of molecules in some regions of deep potential. Similar conclusions have been made by June et al. for methane adsorbed at low temperature in silicalite. ${ }^{7}$

For higher loadings, the decrease of the selfdiffusion coefficient is due to molecular interactions, the QENS results reflecting the overall average contribution of the molecules. The self-diffusion coefficients are, as in the case of ethane, within a factor 2 of those measured by n.m.r. ${ }^{13}$ and within a factor 5 of those determined by the frequency-response method ${ }^{3,4}$ However, these data differ by $\approx 2$ orders of magnitude from those obtained with a molecular

Table 2 Values of the parameters for the translational and rotational motions of propane adsorbed at different loadings in NaZSM-5, at $300 \mathrm{~K}$ (values in parentheses correspond to n.m.r. results ${ }^{13}$ )

\begin{tabular}{llll}
\hline & 1.5 molecule/u.c. & 4 molecules/u.c. & 8 molecules/u.c. \\
\hline$D\left(\mathrm{~m}^{2} \mathrm{~s}^{-1}\right)$ & $1.2 \times 10^{-9}$ & $1.3 \times 10^{-9}$ & $6 \times 10^{-10}$ \\
& & $\left(2.8 \times 10^{-9}\right)$ & $\left(5 \times 10^{-10}\right)$ \\
$\tau_{0}(\mathrm{~s})$ & $1.18 \times 10^{-10}$ & $0.9 \times 10^{-10}$ & $1.2 \times 10^{-10}$ \\
$\left\langle\left.\right|^{2}\right\rangle^{1 / 2}(A)$ & 9.2 & 8.4 & 6.6 \\
$\left\langle d^{2}\right\rangle\left(\AA^{2}\right)$ & 0.53 & 0.43 & 0.31 \\
$r(A)$ & 2 & 2 & 1.83 \\
$D_{r}\left(\mathrm{~s}^{-1}\right)$ & $1.35 \times 10^{11}$ & $2.2 \times 10^{11}$ & $3.3 \times 10^{11}$ \\
\hline
\end{tabular}

Table 3 Values of the parameters for the translational and rotational motions of propane adsorbed at different temperatures in NaZSM-5, for a loading of 3 molecules/u.c.

\begin{tabular}{llll}
\hline & \multicolumn{1}{c}{$300 \mathrm{~K}$} & \multicolumn{1}{c}{$373 \mathrm{~K}$} & \multicolumn{1}{c}{$453 \mathrm{~K}$} \\
\hline$D\left(\mathrm{~m}^{2} \mathrm{~s}^{-1}\right)$ & $1.25 \times 10^{-9}$ & $1.85 \times 10^{-9}$ & $2.43 \times 10^{-9}$ \\
$\tau_{0}(\mathrm{~s})$ & $0.95 \times 10^{-10}$ & $0.56 \times 10^{-10}$ & $0.45 \times 10^{-10}$ \\
$\left\langle 1^{2}\right\rangle^{1 / 2}(\AA)$ & 8.5 & 7.9 & 8.1 \\
$<d^{2}>\left(\AA^{2}\right)$ & 0.45 & 0.32 & 0.62 \\
$r(\AA)$ & 2 & 2.16 & 2.56 \\
$D_{r}\left(\mathrm{~s}^{-1}\right)$ & $2 \times 10^{11}$ & $3.7 \times 10^{11}$ & $1.7 \times 10^{11}$ \\
\hline
\end{tabular}

sieve membrane ${ }^{14}$ or zero-length column (ZLC) method. ${ }^{15}$ Possible reasons for these discrepancies are that QENS and n.m.r. techniques measure intracrystalline self-diffusivities, whereas macroscopic experiments can be complicated by intercrystalline mass transport, sorption heat release, and surface barriers. It has been found recently that the use of large crystals dispersed over a wide area minimized sorbent-bed depth effects. ${ }^{4}$

The radii of gyration, $r$, obtained for the uniaxial rotation of propane are close to $2 \AA$, at $300 \mathrm{~K}$. The rotational-diffusion constants, $D_{r}$, are of the same order of magnitude as for ethane.

To derive an activation energy for the selfdiffusion coefficient of propane, other experiments were performed at 300,373 , and $453 \mathrm{~K}$, for one loading ( 3 molecules per u.c.). The values of the refined parameters are given in Table 3. It is found that the mean jump lengths are independent of the temperature, within experimental error; this was also observed by n.m.r. by Caro et al. for alkanes in silicalite. ${ }^{13}$ An Arrhenius plot of the self-diffusion coefficients is shown in Figure 6. An activation energy for the intracrystalline self-diffusion of propane of 5 $\mathrm{kJ} \mathrm{mol} \mathrm{m}^{-1}$ is derived, which is similar to the one previously obtained for methane. ${ }^{2}$

\section{CONGLUSIONS}

The translational and rotational dynamics of ethane and propane adsorbed at different loadings in ZSM-5 zeolite have been studied by quasi-elastic neutron scattering. The translational motion has been interpreted with a jump diffusion model, the mean jump lengths being of the order of $10 \AA$ at low loadings for the two alkanes. The intracrystalline self-diffusion coefficients derived from neutron scattering are found to be very close to those determined by the n.m.r. and frequency-response techniques. However, these values are about 2 orders of magnitude higher than those obtained with a molecular sieve membrane or with the ZLC method.

The activation energy derived by QENS for the self-diffusion coefficient of propane is of $5 \mathrm{~kJ} \mathrm{~mol}^{-1}$ which is similar to the one previously measured for methane in the same zeolite.

Results obtained on the mobility of $n$-butane and $n$-hexane in the same zeolite will be presented in a forthcoming paper. 


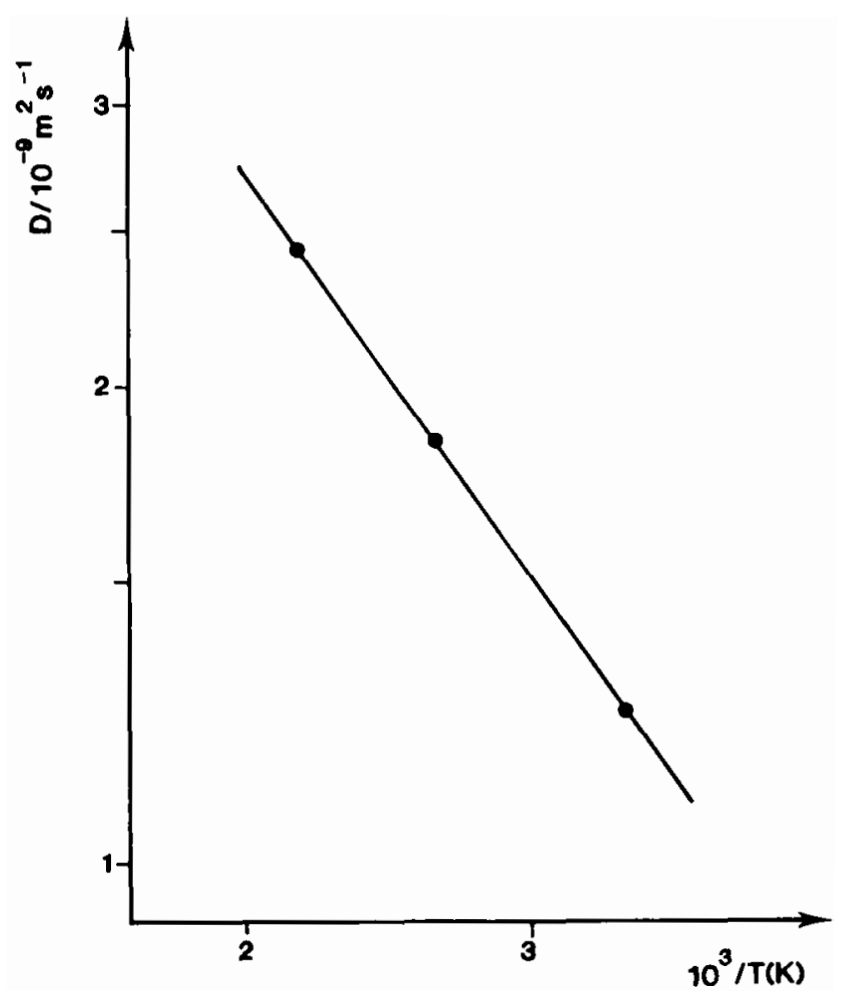

Figure 6 Arrhenius plot of the self-diffusion coefficient of propane in ZSM-5 for a loading of 3 molecules per u.c.

\section{ACKNOWLEDGEMENTS}

We thank G. Clugnet for assistance during the preparation of the samples and J.F. Dutel for measuring the adsorption isotherms.

\section{REFERENGES}

1 Kokotailo, G.T., Lawton, S.L., Olson, D.H. and Meier, W.M. Nature 1978, 272, 437

2 Jobic, H., Bée, M., Caro, J., Bülow, M. and Kärger, J. J. Chem. Soc., Faraday Trans. 1 1989, 85, 4201

3 Van-Den-Begin, N.G. and Rees, L.V.C., in Zeolites: Facts,
Figures, Future (Eds. P.A. Jacobs and R.A. van Santen) Elsevier, Amsterdam, 1989, p. 915

4 Van-Den-Begin, N., Rees, L.V.C., Caro, J. and Bülow, M. Zeolites 1989, 9, 287

5 Demontis, P., Fois, E.S., Suffritti, G.B. and Quartieri, S. J. Phys. Chem. 1990, 94, 4329

6 Pickett, S.D., Nowak, A.K., Thomas, J.M., Peterson, B.K. Swift, J.F.P., Cheetham, A.K., den Ouden, C.J.J., Smit, B. and Post, M.F.M. J. Phys. Chem. 1990, 94, 1233

7 June, R.L., Bell, A.T. and Theodorou, D.N. J. Phys. Chem. 1990, 94, 8232

8 Catlow, C.R.A., Freeman, C.M., Vessal, B., Tomlinson, S.M. and Leslie, M. J. Chem. Soc., Faraday Trans. 1991, 87, 1947

9 Goodbody, S.J., Watanabe, K., MacGowan, D., Walton, J.P.R.B. and Quirke,N. J. Chem. Soc., Faraday Trans. 1991, 87, 1951

10 Nowak, A.K., den Ouden, C.J.J., Pickett, S.D., Smit, B., Cheetham, A.K., Post, M.F.M. and Thomas, J.M. J. Phys. Chem. 1991, 95, 848

11 Förste, Chr., Heink, W., Kärger, J., Pfeifer, H., Feokistova, N.N. and Zhdanov, S.P. Zeolites 1989, 9, 299

12 Jobic, H., Bée, M. and Dianoux, A.J. J. Chem. Soc., Faraday Trans. 1 1989, 85, 2525

13 Caro, J., Bülow, M., Schirmer, W., Kärger, J., Heink, W., Pfeifer, H., Zdanov, S.P. J. Chem. Soc., Faraday Trans. 1 $1985,81,2541$

14 Hayhurst, D.T. and Paravar, A.R. Zeolites 1988, 8, 27

15 Eic, M. and Ruthven, D.M., in Zeolites: Facts, Figures, Future (Eds. P.A. Jacobs and van Santen R.A.) Elsevier, Amsterdam, 1989, p. 897

16 Jobic, H., Bée, M. and Kearley, G.J. Zeolites 1989, 9, 312

17 Willis, B.T.M., Ed. Chemical Applications of Thermal Neutron Scattering, Oxford University Press, London 1973

18 Jobic, H., Bée, M., Caro, J., Bülow, M., Kärger, J. and Pfeifer, $\mathrm{H}$., in Studies in Surface Science and Catalysis (Eds. G. Ohlmann, H. Pfeifer and R. Fricke) Elsevier, Amsterdam, 1991 , vol. 65 , p. 445

19 Sears, V.F. Can. J. Phys. 1966, 44, 1299; 1967, 45, 234

20 Dianoux, A.J., Volino, F. and Hervet, H. Mol. Phys. 1975, 30, 1181

21 Jobic, H., Renouprez, A., Bée, M. and Poinsignon, C. J. Phys. Chem. 1986, 90, 1059

22 Jobic, H., Bée, M., Kärger, J., Pfeifer, H. and Caro, J. J. Chem. Soc., Chem. Commun. 1990, 341

23 Singwi, K.S. and Sjölander, A. Phys. Rev. 1960, 119, 863

24 June, R.L., Bell, A.T. and Theodorou, D.N. J. Phys. Chem. $1990,94,1508$

25 Jobic, H. Chem. Phys. Lett. 1990, 170, 217

26 Bée, M., Quasielastic Neutron Scattering, Adam Hilger, Bristol, 1988

27 Snyder, R.G. and Schachtschneider, J.H. Spectrochim. Acta 1965, 21, 169 